\title{
A GENERALIZATION OF OUTER PARALLEL SETS OF A CONVEX SET
}

\author{
J. R. SANGWINE-YAGER
}

(Communicated by Christopher Croke)

\begin{abstract}
An outer parallel set is formed by adding outer normals of fixed length to the boundary points of a convex set. In this paper the length of the outer normals varies as a function of the direction. This construction yields a geometric interpretation of the dual quermassintegrals of Lutwak and bounds on certain mixed volumes. An inequality of Firey will follow from one of the mixed volume bounds.
\end{abstract}

\section{INTRODUCTION}

Let $K$ be a convex set with nonempty interior in $d$-dimensional Euclidean space $E^{d}$. The unit ball centered at the origin will be denoted by $B$, and $S^{d-1}$ is the unit sphere. The outer parallel set of $K$ at distance $t>0$ is $K_{t}=\{k+t b \mid k \in K$ and $b \in B\}$. These sets may be used to define the quermassintegrals $W_{i}, i=0, \ldots, d$, from the classical Steiner's formula

$$
V\left(K_{t}\right)=\sum_{i=0}^{d}\left(\begin{array}{l}
d \\
i
\end{array}\right) t^{i} W_{i}(K)
$$

Here $V=W_{0}$ is $d$-dimensional volume, $d W_{1}$ is $(d-1)$-dimensional surface area, and $W_{d}$ is constant and equal to $V(B)$. The outer parallel set at distance $t$ may also be constructed by adding to each boundary point of $K$ vectors of length $t$ which are outer normals to the boundary of $K$ at that point. It is this view of the outer parallel sets which we will generalize.

Let $f$ be a nonnegative measurable function on $S^{d-1}$ for which the integrals in (2) are finite. At each boundary point of $K$ at which $u$ is an outer normal add $\theta f(u) u, 0<\theta \leq t, t>0$. The resultant (probably nonconvex) set will be called a generalized outer parallel set of $K$ at distance $t$ denoted by $K_{t f(u)}$. It follows from a result of Weil [10] that

$$
V\left(K_{t f(u)}\right)=V(K)+\frac{1}{d} \sum_{i=1}^{d}\left(\begin{array}{l}
d \\
i
\end{array}\right) t^{i} \int_{S^{d}-1} f(u)^{i} d S_{d-i}(K, u),
$$

Received by the editors March 8, 1993 and, in revised form, August 25, 1993.

1991 Mathematics Subject Classification. Primary 52A40, 52A20.

Key words and phrases. Brush set, mixed volume, outer parallel set, polar dual, quermassintegral. 
where the $S_{i}$ are the area measures. We will use (2) to obtain a geometric interpretation of the dual mixed volumes of Lutwak [4] and to obtain bounds on certain mixed volumes of convex sets. One of these bounds will lead to an inequality of Firey [2].

\section{Preliminaries}

The scalar product in $E^{d}$ is represented by $\langle\cdot, \cdot\rangle$, its induced norm by $\|\cdot\|$, and " + " is Minkowski or vector addition. The boundary of a convex set $K$ is denoted by bd $K$. The support function is a real-valued function on $E^{d}$ defined by $h(K, x)=\max \{\langle x, y\rangle: y \in K\}$. For all convex sets $K$ and $L$, positive scalars $s$ and $t$, and $x \in E^{d}, h(s K+t L, x)=\operatorname{sh}(K, x)+t h(L, x)$. The radial function is a real-valued function on $S^{d-1}$ defined for a convex set $K$ with the origin in its interior by $\rho(K, u)=\max \{\lambda>0: \lambda u \in K\}$. For all convex sets $K$ and $L$ with the origin in their interiors, positive scalars $s$ and $t$, and $u \in S^{d-1}, \rho(s K, u)=s \rho(K, u)$ and $\rho(s K+t L, u) \geq s \rho(K, u)+t \rho(L, u)$. The polar dual of a convex set $K$ with the origin in its interior is defined by

$$
K^{*}=\left\{x \in E^{d}:\langle x, y\rangle \leq 1, \text { for all } y \in K\right\} .
$$

The only properties of the polar dual which will be needed in this work are

$$
h\left(K^{*}, u\right)=\rho(K, u)^{-1} \quad \text { and } \quad \rho\left(K^{*}, u\right)=h(K, u)^{-1} .
$$

The mixed volume of $d$ convex sets in $E^{d}$ is defined within a theorem of Minkowski which generalizes the Steiner formula (1). The theorem states that the volume of the linear combination $t_{1} K_{1}+\cdots+t_{m} K_{m}$, where $K_{1}, \ldots, K_{m}$ are convex sets and $t_{1}, \ldots, t_{m}$ are positive scalars, is a $d$ th degree homogeneous polynomial in $t_{1}, \ldots, t_{m}$, that is,

$$
V\left(\sum_{i=1}^{m} t_{i} K_{i}\right)=\sum_{i_{1}=1}^{m} \cdots \sum_{i_{d}=1}^{m} t_{i_{1}} \cdots t_{i_{d}} V\left(K_{i_{1}}, \ldots, K_{i_{d}}\right) .
$$

The coefficients are chosen to be invariant under permutations of their arguments and are called the mixed volumes. The mixed volumes and quermassintegrals are related by the identity

$$
V(\underbrace{K, \ldots, K}_{i}, \underbrace{B, \ldots, B}_{d-i})=W_{d-i}(K), \quad i=1, \ldots, d .
$$

The name quermassintegral comes from the property that $W_{d-i}(K), 1 \leq i \leq$ $d-1$, is proportional to the mean value of the $i$-dimensional volumes of the projections of $K$ onto $i$-dimensional subspaces (see [1, p. 139]).

An excellent treatment of area measures may be found in [7, §4.2]. Here we present some necessary definitions. For any point $x \in E^{d}$ the unique point of $K$ nearest to $x$ is denoted by $p(K, x)$. If $x \notin K$, then $p(K, x) \in \operatorname{bd} K$ and $x-p(K, x)$ is an outer normal to bd $K$ at $p(K, x)$. For each Borel subset $\omega$ of $S^{d-1}$ we define the brush set of $K$ at a distance $t>0$ by

$$
B_{t}(K, \omega)=\left\{x: 0<\|x-p(K, x)\| \leq t \text { and } \frac{x-p(K, x)}{\|x-p(K, x)\|} \in \omega\right\} .
$$

Each brush set is a Borel set and its Lebesgue measure $V_{t}(K, \omega)$ is a polynomial in $t$ of degree at most $d$ whose constant term is zero. This polynomial is used 
to define the area measures as follows:

$$
V_{t}(K, \omega)=\frac{1}{d} \sum_{i=0}^{d-1}\left(\begin{array}{l}
d \\
i
\end{array}\right) t^{d-i} S_{i}(K, \omega) .
$$

This formula may be viewed as a local version of the Steiner formula (1). If $K$ and $L$ are convex sets in $E^{d}$ with the origin in the interior of $L$, the following identities are required below:

$$
V(L)=\frac{1}{d} \int_{S^{d-1}} \rho(L, u)^{d} d S_{0}(B, u),
$$

where we note that $S_{0}(K, \cdot)=S_{0}(B, \cdot)$, and

$$
V(K, \ldots, K, L)=\frac{1}{d} \int_{S^{d-1}} h(L, \mu) d S_{d-1}(K, u) .
$$

We now give our defintion of generalized outer parallel sets: If $K$ is a convex set in $E^{d}, t>0, f$ is a nonnegative measurable function on $S^{d-1}$ for which the integrals in (2) are finite, and $P=\left\{u \in S^{d-1}: f(u)>0\right\}$, then

$$
K_{t f(u)}=K \cup \bigcup_{u \in P} B_{t f(u)}(K,\{u\}) .
$$

It is evident from simple examples that the generalized outer parallel sets may not be convex. Although it is often the case that the Minkowski sum of two convex sets is not homothetic to a generalized outer parallel set of either, the next lemma provides useful relations. We note that there are other methods of generalizing outer parallel sets; for example, see [7, §6.5].

Lemma. If $K$ and $L$ are convex sets in $E^{d}$ with the origin in the interior of $L$ and $t>0$, then

$$
K_{t \rho(L, u)} \subset K+t L \subset K_{t h(L, u)} .
$$

Proof. It suffices to let $t=1$. The first inclusion follows directly from the fact that $\rho(L, u) u \in L$ for all $u \in S^{d-1}$. For the second inclusion let $x=k+l$, for any $k \in K$ and $l \in L$. If $x \in K$, we are done. If not, $p=p(K, x)$ is a boundary point of $K$ and $u=(x-p) /\|x-p\|$ is an outer normal to $K$ at $p$. We must show that $\|x-p\| \leq h(L, u)$. Since $\langle k, u\rangle \leq\langle p, u\rangle$, we have

$$
\|x-p\|=\langle x-p, u\rangle=\langle l, u\rangle+\langle k-p, u\rangle \leq\langle l, u\rangle \leq h(L, u) .
$$

In the parallel set $K_{t f(u)}$ all outer normals to $K$ in the direction $u$ are extended the same distance. An alternate construction is to extend all outer normals at a given boundary point of $K$ the same distance. If $f$ is a nonnegative measurable function on bd $K$ for which the integrals in (8) are finite, $t>0$, and $P=\{p \in$ bd $K: f(p)>0\}$, then let

$$
K_{t f(p)}=K \cup \bigcup_{p \in P}\{x: 0<\|x-p(K, x)\| \leq t f(p) \text { and } p(K, x)=p\} .
$$

In the result analogous to (2) (see (8)) the integration is with respect to Federer's curvature measures. 


\section{Proof of (2) AND APPLICATIONS}

Equation (2) follows from a more general result proved by Weil [10, p. 99]; this result first appears in Weil [9, (4.7)]. In the notation of the latter citation we have

$$
\begin{aligned}
\int_{K \cap E_{q}}=\varnothing & g\left(s\left[K, E_{q}\right], u\left[K, E_{q}\right]\right) d \mu_{q}\left(E_{q}\right) \\
= & (d-q) \alpha(d-q) \sum_{j=0}^{d-q-1} \int_{0}^{\infty} \int_{S^{d-1}} s^{d-q-j-1} g(s, u) d S_{j}(K, u) d s,
\end{aligned}
$$

where $E_{q}$ is an element of the homogeneous space of $q$-flats in $E^{d}$ with invariant measure $\mu_{q}, q=0, \ldots, d-1 ; g$ is a measurable function on $(0, \infty) \times S^{d-1}$ for which the integrals above are finite; $s\left[K, E_{q}\right]$ is the shortest distance from $K$ to the $q$-flat and $u\left[K, E_{q}\right] \in S_{d-1}$ is the direction in which that distance is realized; and $\alpha(d-q)$ is the volume of the $(d-q)$-dimensional unit ball. To verify (2), let $f$ be the given function and let $q=0$. Notice that if $x=E_{0}$, then $s\left[K, E_{0}\right]=\|x-p(K, x)\|$ and $u\left[K, E_{0}\right]$ is parallel to $x-p(K, x)$. The result will follow if we define $g$ to be an indicator function, that is, $g(s, u)=1$ if $s \leq f(u)$ and 0 otherwise.

We have a result analogous to (2) when $f$ is a function defined on bd $K$; this follows from Weil [9, (6.3)]. If $K$ is a convex set in $E^{d}$ and $f$ is a nonnegative measurable function on bd $K$ for which the integrals below are finite, then

$$
V\left(K_{t f(p)}\right)=V(K)+\frac{1}{d} \sum_{i=1}^{d}\left(\begin{array}{c}
d \\
i
\end{array}\right) t^{i} \int_{\mathrm{bd} K} f(p)^{i} d C_{d-i}(K, p) .
$$

The $C_{i}$ are Federer's curvature measures, and they are also discussed in [7]. As an application of $(8)$, let $f(p)=r(K, p)$ be the interior reach at $p \in \operatorname{bd} K$ where

$$
r(K, p)=\sup \left\{\lambda: p \in x+\lambda B \subset K \text { for some } x \in E^{d}\right\} .
$$

The generalized outer parallel set of $K$ at distance $r(K, p)$ may be viewed as the set $K$ "turned inside out". In Sangwine-Yager [6] the author obtains a representation of $V(K)$ as an alternating sum of the integrals in (8).

Theorem. If $K$ and $L$ are convex sets in $E^{d}$ and the origin is in the interior of $L$, then

$$
\frac{1}{d} \int_{S^{d-1}} \rho(L, u)^{d-1} d S_{1}(K, u) \leq V(K, L, \ldots, L)
$$

and

$$
V(K, \ldots, K, L, L) \leq \frac{1}{d} \int_{S^{d-1}} h(L, u)^{2} d S_{d-2}(K, u) .
$$

Proof. It follows from the lemma and the monotonicity of volume that

$$
V\left(K_{t \rho(L, u)}\right) \leq V(K+t L) \text { and } V(K+t L) \leq V\left(K_{t h(L, u)}\right) \text {. }
$$

By (2) or (4), as appropriate, each of the volumes above may be represented as a polynomial of degree $d$ in $t$ with constant term $V(K)$. In the left-hand inequality (6) implies that the coefficients of $t^{d}$ are equal, and in the right-hand inequality (7) implies that the coefficients of $t$ are equal. Thus the order of the 
polynomials may be reduced to $d-2$. Any inequality for polynomials of the same order results in inequalities between the coefficients of the lowest- and highest-order terms. In the theorem we give only two inequalities because, in both the left- and right-hand cases, the other inequality is well known. They are

$$
\frac{1}{d} \int_{S^{d-1}} \rho(L, u) d S_{d-1}(K, u) \leq V(K, \ldots, K, L)
$$

and

$$
V(L) \leq \frac{1}{d} \int_{S^{d-1}} h(L, u)^{d} d S_{0}(K, u)
$$

and follow from $\rho(L, u) \leq h(L, u)$ and (7) and (6), respectively.

Lutwak [4] defines the dual mixed volume to be

$$
\widetilde{V}\left(K_{1}, \ldots, K_{d}\right)=\frac{1}{d} \int_{S^{d-1}} \rho\left(K_{1}, u\right) \cdots \rho\left(K_{d}, u\right) d S_{0}(B, u),
$$

and the dual quermassintegrals are

$$
\widetilde{W}_{d-i}(K)=\widetilde{V}(\underbrace{K, \ldots, K}_{i}, \underbrace{B, \ldots, B}_{d-i}), \quad i=1, \ldots, d .
$$

The dual mixed volumes share many properties with classical mixed volumes; for example, there are analogues to (1) and (4) where vector addition is replaced with radial addition. See [5] and [1, pp. 158-159] for further properties. In the theory of dual mixed volumes the integration is always with respect to $S_{0}(B, \cdot)$. To use (2) to obtain a geometric interpretation of the dual quermassintegrals, first note that in a variation of (1) the classical quermassintegrals are the coefficients of the volume polynomial for the outer parallel sets $B+t K$. If we let $K=B$ and $f(u)=\rho(K, u)$ in (2), then the dual quermassintegrals are the corresponding coefficients of the volume polynomial for the generalized outer parallel sets $B_{t \rho(K, u)}$. Unfortunately it does not seem possible to extend this interpretation to the dual mixed volumes.

An inequality of Lutwak [4] states that $\widetilde{V}\left(K_{1}, \ldots, K_{d}\right) \leq V\left(K_{1}, \ldots, K_{d}\right)$. In the case $K_{1}=\cdots=K_{d-1}=L$ and $K_{d}=K \neq B$, neither Lutwak's inequality nor the first inequality of the theorem provide a universally stronger lower bound for the mixed volume. For example, if $d=2$ and $K=L$, then equality holds in Lutwak's inequality but not in general in the theorem. The situation is reversed if $d=2$ and $L=B$.

Finally, if $K$ is a convex set with the origin as an interior point and we apply (3) to the theorem for $L=K^{*}$, then

$$
\frac{1}{d} \int_{S^{d-1}} h(K, u)^{1-d} d S_{1}(K, u) \leq V\left(K, K^{*}, \ldots, K^{*}\right)
$$

and

$$
V\left(K, \ldots, K, K^{*}, K^{*}\right) \leq \frac{1}{d} \int_{S^{d-1}} \rho(K, u)^{-2} d S_{d-2}(K, u) .
$$

When Jensen's inequality and (5) are applied to (9) we have

$$
W_{d-1}(K)^{d} \leq W_{d-2}(K)^{d-1} V\left(K, K^{*}, \ldots, K^{*}\right) .
$$

This result for $d=2$ and $i=1$ may be combined with the isoperimetric inequality in the plane, $\pi V(K) \leq V(K, B)^{2}$, to yield

$$
\pi \leq V\left(K, K^{*}\right),
$$


which is a well-known inequality due to Steinhardt [8] and Firey [2]. We also note that for $d=2,(10)$ is a special case of an inequality of Ghandehari [3, (25)].

\section{ACKNOWLEDGMENT}

The author is indebted to Professors Wm. J. Firey and E. Lutwak for contributions to this paper.

\section{REFERENCES}

1. Ju. D. Burago and V. A. Zalgaller, Geometric inequalities, Springer, Berlin, 1988.

2. W. Firey, The mixed area of a convex body and its polar reciprocal, Israel J. Math 1 (1963), 201-202.

3. M. Ghandehari, Polar duals of convex bodies, Proc. Amer. Math. Soc. 113 (1991), 799-808.

4. E. Lutwak, Dual mixed volumes, Pacific J. Math. 58 (1975), 531-538.

5. __ Intersection bodies and dual mixed volumes, Adv. Math. 69 (1988), 232-261.

6. J. R. Sangwine-Yager, A representation of volume involving interior reach, Math. Ann. 298 (1994), $1-5$.

7. R. Schneider, Convex bodies: the Brunn-Minkowski theory, Cambridge Univ. Press, Cambridge, 1993.

8. F. Steinhardt, On distance functions and on polar series of convex bodies, Ph.D. Thesis, Columbia Univ., 1951.

9. W. Weil, Kinematic integral formulas for convex bodies, Contributions to Geometry (J. Tolke and J. M. Wills, eds.), Birkhäuser, Basel, 1979, pp. 60-76.

10. _ Zufälige Berührung konvexer Körper durch q-dimensionale Eben, Resultate Math. 4 (1981), 84-101.

Mathematical Sciences, Saint Mary's College, Moraga, California 94575

E-mail address: sangwine@galileo.stmarys-ca.edu 